\title{
Enhanced ferromagnetism induced by structural phase transitions in $\mathrm{Co}_{2} \mathrm{As}_{1-x} \mathrm{P}_{x}$
}

\author{
Chih-Wei Chen, Jiakui K. Wang, and Emilia Morosan \\ Department of Physics and Astronomy, Rice University, Houston, TX 77005 USA
}

(Dated: November 12, 2015)

\begin{abstract}
$\mathrm{P}$ doping in $\mathrm{Co}_{2} \mathrm{As}$ induces two structural transitions, resulting in an enhanced ferromagnetic state at intermediate $\mathrm{P}$ compositions. In $\mathrm{Co}_{2} \mathrm{As}_{1-x} \mathrm{P}_{x}$, doping induces a room temperature $\alpha$-to$\beta$ structural distortion around $x=0.04$, similar to what temperature $(\mathrm{T}=725 \mathrm{~K})$ does in the parent compound $(x=0)$. The resulting $\beta$ phase displays an enhanced ferromagnetic ground state. Close to $x=0.85$, a hexagonal-to-orthorhombic phase transition occurs, concomitant with the quenching of the magnetic order. Band structure calculations for the three different phases confirm the experimental observations while revealing remarkably high charge carrier polarization rate for the $\beta$ phase.

PACS numbers: 75.50.Cc, 75.10.Lp, 75.30.-m
\end{abstract}

\section{INTRODUCTION}

Binary $M_{2} P n$ or ternary $M M^{\prime} P n$ compounds, where $M, M^{\prime}=$ transition metals and $P n=$ pnictogen, have attracted intensive interest due to their strong electron correlations and diverse ground states, including magnetism and superconductivity. ${ }^{1-10}$ Doping is often associated with structural phase transitions, which, in turn, drive changes to the physical ground state. ${ }^{3,4,9,11} \mathrm{Co}_{2}$ As is an example of a $M_{2} P n$ hexagonal compound, for which a structural phase transition takes place at $T=725 \mathrm{~K}$, resulting in an $\alpha$ low temperature phase (Fig. 1a) and a $\beta$ high temperature phase (Fig. 1b). ${ }^{2}$ Previously reported magnetization measurements show Curie-Weiss-like behavior for $T>200 \mathrm{~K}$, with Weiss temperatures $\theta=-680 \mathrm{~K}$ for the $\alpha$ phase and $-430 \mathrm{~K}$ for the $\beta$ phase. $^{2}$ Even though these negative $\theta$ values suggested antiferromagnetic (AFM) coupling, the extrapolated susceptibility was suggested to be divergent around 10 $\mathrm{K}$, which would indicate ferromagnetic (FM) order in the $\alpha$ phase. ${ }^{2}$ However, these conclusions were reached in the absence of low temperature magnetization data (lower than the $80 \mathrm{~K}^{2}$ ) which is needed to study the ground state.

In this study, we show that, at room temperature, $\mathrm{P}$ doping in $\mathrm{Co}_{2} \mathrm{As}_{1-x} \mathrm{P}_{x}$ induces the $\alpha$-to- $\beta$ structural phase transition around $x=0.04$, as well as a hexagonal-to-orthorhombic phase transition for $x>0.85$. After accounting for temperature-independent contributions to the magnetic susceptibility $\chi_{0}$, magnetization measurements on $\mathrm{Co}_{2} \mathrm{As}_{1-x} \mathrm{P}_{x}$ down to $T=2 \mathrm{~K}$ (lower than the $80 \mathrm{~K}$ in the previous study ${ }^{2}$ ) reveal both positive $\theta$ values and divergent susceptibility, consistent with FM ground state for the hexagonal phase $(0 \leq x \leq 0.85)$. Remarkably, the $\alpha$-to- $\beta$ structural phase transition at $x=0.04$ enhances the ferromagnetism in the $\beta$ phase compared to the $\alpha$ phase. Moreover, the subsequent phase transition at $x>0.85$ quenches the magnetic ground state in the orthorhombic phase. In the orthorhombic phase $(0.95 \leq x \leq 1)$, the magnetic susceptibility is temperature-independent when $T>50 \mathrm{~K}$, consistent with Pauli paramagnetism. The experimental results suggest strong correlations between the crystal structure and the magnetic ground state in $\mathrm{Co}_{2} \mathrm{As}_{1-x} \mathrm{P}_{x}$. Band structure calculations for the $\alpha$ and $\beta$ hexagonal phases, as well as for the orthorhombic phase, confirmed the enhancement of the ferromagnetism in the $\beta-\mathrm{Co}_{2} \mathrm{As}_{1-x} \mathrm{P}_{x}$ phase. 


\section{METHODS}

Polycrystalline $\mathrm{Co}_{2} \mathrm{As}_{1-x} \mathrm{P}_{x}(0 \leq x \leq 1)$ samples were prepared by solid state reaction, in which ground powders of Co (Alfa Aesar, $99.9 \%$ ), As (Alfa Aesar, 99.99, \%) and P (Alfa Aesar, 99.999 \%) were mixed in the ratio of $\mathrm{Co}$ : As : $\mathrm{P}=1.95: 1-x: x$ and sealed in evacuated quartz tubes. The samples were heated between $400^{\circ} \mathrm{C}$ and $900^{\circ} \mathrm{C}$ for six days, with intermediate grindings. Room temperature powder x-ray diffraction (XRD) measurements were performed on a Rigaku D/Max diffractometer with $\mathrm{Cu} \mathrm{K \alpha}$ radiation and a graphite monochromator. Rietveld analysis was done using the GSAS suite of programs. ${ }^{12}$ Temperature- and field-dependent magnetization data were collected in a Quantum Design (QD) Magnetic Property Measurement System (MPMS) for temperatures between 2 $\mathrm{K}$ and $300 \mathrm{~K}$ and applied magnetic field $H$ up to $7 \mathrm{~T}$. DC resistivity measurements were performed in a QD Physical Property Measurement System (PPMS) using a standard four-probe method. Specific heat measurements were also performed in the QD PPMS using an adiabatic relaxation technique.

Band structure calculations within the density functional framework were performed using a full potential linear augmented plane wave method (FP-LAPW), as implemented in the WIEN2k code. ${ }^{13}$ A cutoff parameter $R K_{\max }=7.0$ was used, and the exchange-correlation potential was taken in the commonly used Perdew-Burke-Ernzerhof (PBE) form $^{14}$ of the generalized-gradient approximation. The linear tetrahedron method was employed for the Brillouin zone (BZ) integration, with the $k$-mesh of $10 \times 10 \times 10$ points in the primitive BZ. Both paramagnetic (PM) and ferromagnetic (FM) density of states (DOS) are calculated for $\alpha-\mathrm{Co}_{2} \mathrm{As}, \beta-\mathrm{Co}_{2} \mathrm{As}$ and $\mathrm{Co}_{2} \mathrm{P}$.

\section{EXPERIMENTAL RESULTS}
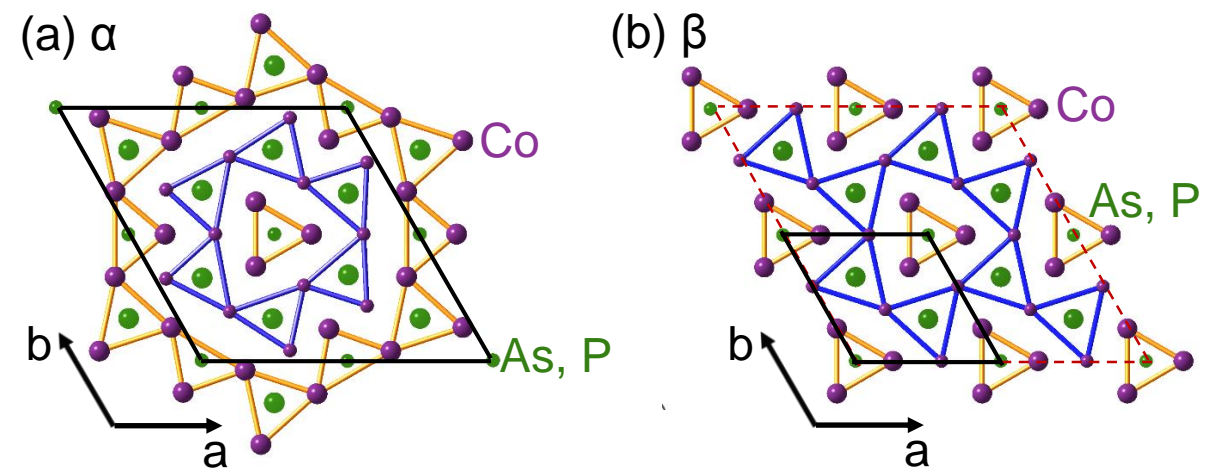

(c) orthorhombic

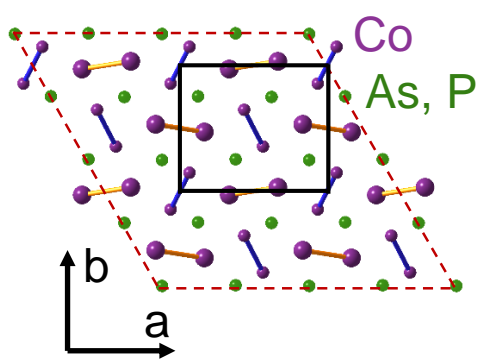

FIG. 1: $\mathrm{Co}_{2} \mathrm{As}_{1-x} \mathrm{P}_{x}$ crystal structure for (a) the hexagonal $\alpha$ phase, (b) the hexagonal $\beta$ phase, and (c) the orthorhombic phase. The unit cell is indicated by the black solid line, and the purple dashed parallelograms are used as a comparison with the hexagonal $\alpha$ phase.

The $\mathrm{Co}_{2}$ As crystal structure consists of different Co (As) sites, six (five) in the hexagonal $\alpha$ phase, two (two) in the hexagonal $\beta$ phase, and two (one) in the orthorhombic phase), with the atoms in the $\mathrm{z}=0(0.5)$ plane represented by the larger (smaller) spheres in Fig. 1. The hexagonal structure with space group $P 6 \overline{2} m^{11}$ persists up to 1313 $\mathrm{K}$, with a structural distorsion around $725 \mathrm{~K}$. In the low temperature $\alpha$ phase $(\mathrm{T} \leq 725 \mathrm{~K}$, Fig. 1a), the Co atoms in different planes form rings of distorted triangles, centered around As atoms in planes half a unit cell above and below. At $T=725 \mathrm{~K}$, the Co atoms move to higher symmetry positions (Fig. 1b), resulting in the high temperature 

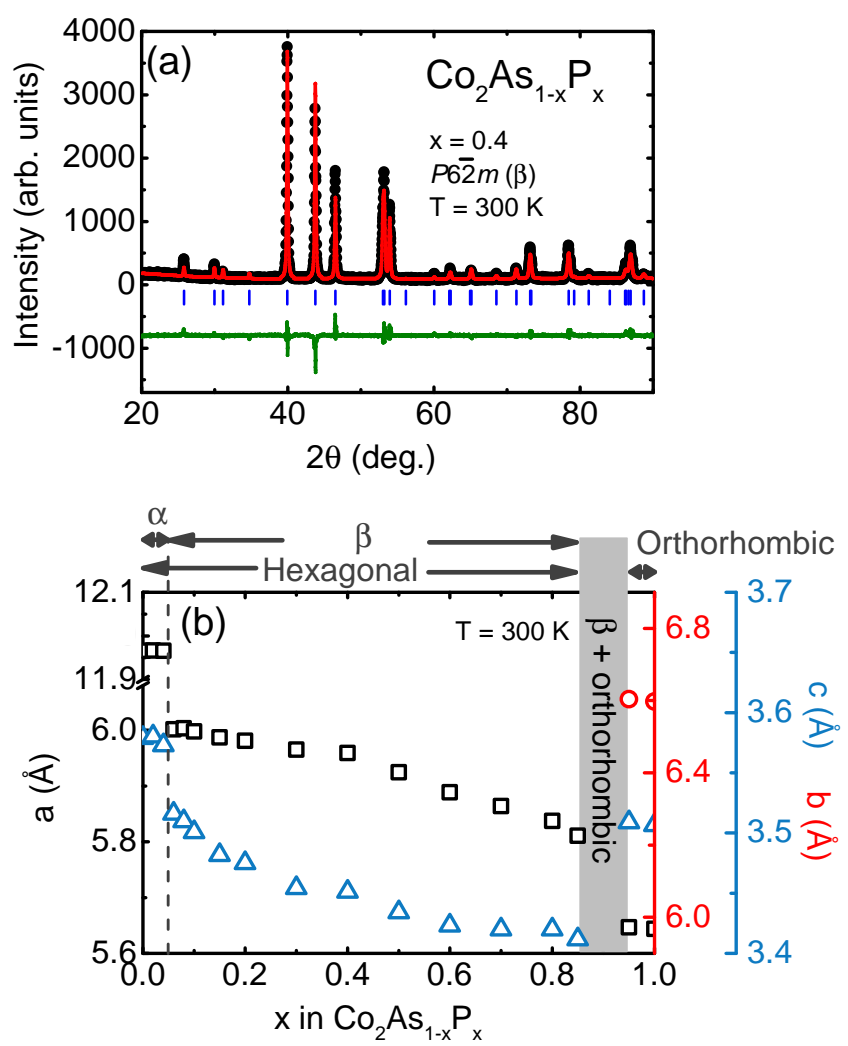

FIG. 2: a Rietveld refinement profile for the room temperature XRD data for $\mathrm{Co}_{2} \mathrm{As}_{0.6} \mathrm{P}_{0.4}$. The difference between measured data (black) and Rietveld fit (red) is shown as a green line. The calculated Bragg peak positions for $\mathrm{Co}_{2} \mathrm{As}_{0.6} \mathrm{P}_{0.4}$ are indicated by blue vertical markers. (b) Lattice parameters of $\mathrm{Co}_{2} \mathrm{Ass}_{1-x} \mathrm{P}_{x}$ as a function of $\mathrm{x}$. Error bars are smaller than the symbol size.

hexagonal $\beta$ phase. While this crystallographic modification preserves the same space group, the $a$ and $b$ unit cell parameters (outlined in black) are nearly half when compared to those of the $\alpha$ phase. ${ }^{2}$

This high temperature $\beta$ phase appears to also be stabilized at room temperature by $\mathrm{P}$ doping. $\mathrm{P}$ doping changes the room temperature crystal structure of $\mathrm{Co}_{2} \mathrm{As}_{1-x} \mathrm{P}_{x}$ from the low temperature $\alpha$ phase to the high temperature $\beta$ phase when $0.06 \leq x \leq 0.85$, while the $\alpha$ phase is preserved for lower $x$ values. Further $\mathrm{P}$ doping induces a structural phase transition from the hexagonal P6 $2 \bar{m}$ to an orthorhombic structure with space group Pnam (Fig. 1c) for $0.95 \leq x \leq 1 . \mathrm{Co}_{2} \mathrm{As}_{1-x} \mathrm{P}_{x}$ forms mixed phases and no single phase was observed for $0.85<x<0.95$. The Rietveld refinement of the $\mathrm{Co}_{2} \mathrm{As}_{1-x} \mathrm{P}_{x}$ room temperature powder XRD patterns, with an example shown in Fig. 2a for $x=0.40$, confirms the structure and purity. The lattice parameters for $\mathrm{Co}_{2} \mathrm{As}_{1-x} \mathrm{P}_{x}$ as a function of $x$ are shown in Fig. 2b, and are close to previously reported values. ${ }^{4,11}$ Initially, $\mathrm{P}$ doping in $\mathrm{Co}_{2} \mathrm{As}_{1-x} \mathrm{P}_{x}$ leaves the lattice parameters virtually unchanged in the $\alpha$ phase, for $x \leq 0.04$. An $\alpha$-to- $\beta$ structural transition occurs between $x=0.04$ and 0.06 , with both the $a$ (squares, left axis) and $c$ (triangles, right axis) lattice parameters changing abruptly (Fig. 2b). Upon further increasing the amount of $\mathrm{P}$ in place of the larger As atoms, a monotonic decrease of the lattice parameters is observed for $0.06 \leq x \leq 0.85$ (Fig. 1b), consistent with the Vegard's law. ${ }^{15}$ The hexagonal-to-orthorhombic transition close to $x=0.85$ also results in an abrupt change in all lattice parameters: as $x$ increases from $x=0.85$ to 0.95, $a$ decreases, with $b$ (circles, right axis) slightly larger than $a$, and $c$ increases. 


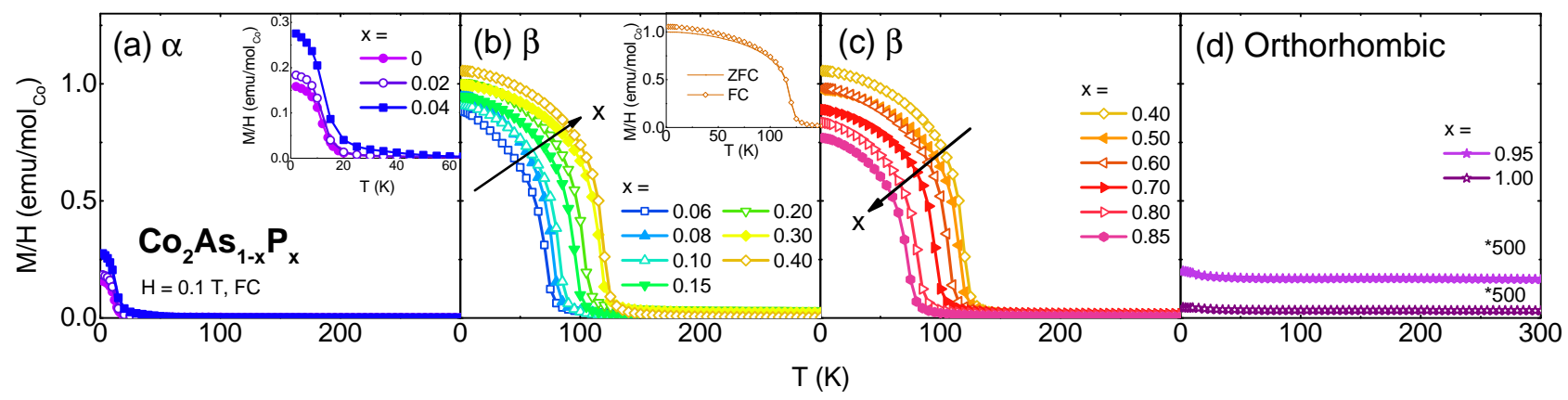

FIG. 3: Temperature-dependent susceptibility for (a) $0 \leq x \leq 0.04(\alpha)$, (b) $0.06 \leq x \leq 0.4(\beta)$, (c) $0.4 \leq x \leq 0.85$ ( $\beta$ ), and (d) $0.95 \leq x \leq 1$ (orthorhombic). The inset in (a) shows the low-temperature susceptibility range for clarity. The inset in (b) shows the ZFC/FC split of the susceptibility of the $x=0.4$ compound, which has the maximum split in these samples.

It appears that both structural transitions have drastic effects on the magnetism in $\mathrm{Co}_{2} \mathrm{As}_{1-x} \mathrm{P}_{x}$, resulting in enhanced magnetic moments and ferromagnetic ordering temperatures in the $\beta$ phase. The magnetization in the $\alpha$ phase $(x \leq 0.04)$ increases rapidly on cooling when the temperature drops below $\sim 10 \mathrm{~K}$ (Fig. 3a), reaching about $0.25 \mathrm{emu} / \mathrm{mol}_{\mathrm{Co}}$ at $T=2 \mathrm{~K}$. However, for $x=0.06$, a nearly four times increase in the $T=2 \mathrm{~K}$ magnetization occurs following the ferromagnetic ordering below $\sim 70 \mathrm{~K}$, concomitant with the $\alpha$-to- $\beta$ structural phase transition (Fig. $3 \mathrm{~b}$ ). The ordering temperature increases with $\mathrm{P}$ doping and it reaches a maximum around $120 \mathrm{~K}$ at $x=0.4$. Further $\mathrm{P}$ doping in the $\beta$ phase decreases this temperature such that it drops again close to $70 \mathrm{~K}$ at $x=0.85$ (Fig. 3c). Following the composition range of mixed phases for $0.85<\mathrm{x}<0.95$ (gray area in Fig. 2b), consistent with the previous report, ${ }^{4}$ the ferromagnetic order is completely suppressed. The magnetic susceptibility of the orthorhombic $\mathrm{Co}_{2} \mathrm{As}_{1-x} \mathrm{P}_{x}(x \geq 0.95$ in Fig. $3 \mathrm{~d})$ is nearly temperature-independent, consistent with the Pauli susceptibility about two orders of magnitude smaller than the paramagnetic susceptibility of the $\beta$ phase.

In the ferromagnetic region $(0 \leq x \leq 0.85)$, the magnetic susceptibility displays Curie-Weiss-like behavior (Fig. 4). After a temperature-independent contribution $\chi_{0}$ is subtracted, $H /\left(M-M_{0}\right)$ is linear in $T$ above the temperature where the divergence of $M(T)$ takes place (Fig. 3 a-c). The linear fits of the inverse susceptibility in the paramagnetic state yield effective moment values $\mu_{\text {eff }}$ about $0.8 \mu_{B} /$ Co in the $\alpha$ phase, and between $1.59(x$ $=0.06)$ and $1.98(x=0.4) \mu_{\mathrm{B}} /$ Co in the $\beta$ phase. Within the ionic picture, the corresponding theoretical effective moment values of the Co oxidation states are $\mu_{\mathrm{eff}}^{t h}\left(\mathrm{Co}^{2+}\right)=3.87 \mu_{\mathrm{B}}$, and $\mu_{\mathrm{eff}}^{t h}\left(\mathrm{Co}^{3+}\right)=4.90 \mu_{\mathrm{B}}(L=0)$, and even larger for the unquenched orbital moment case $(L>0) .{ }^{16}$ The experimental $\mu_{e f f}^{\text {exp }}$ for $x=0.04$ is $0.8 \mu_{B} /$ Co, several times smaller than the theoretical values of either $\mathrm{Co}^{2+}$ and $\mathrm{Co}^{3+}$. For the $\beta$ phase, even though the $\mu_{e f f}^{\text {exp }}$ values are more than double (1.59 to $\left.1.98(x=0.4) \mu_{\mathrm{B}} / \mathrm{Co}\right)$, they remain much smaller than the theoretical values. The smaller-than-calculated values for $\mu_{\text {eff }}$ may be due to either itinerant moment magnetism or crystal field effects, or both, and this is to be addressed with the field-dependent magnetization data, as well as $H=0$ resistivity and specific heat measurements presented below, together with band structure calculations.

Field-dependent magnetization isotherms $M(H)$ reveal ferromagnetic behavior of both $\alpha$ and $\beta$ phases (Fig. 5ac), with the magnetic order suppressed in the orthorhombic phase. The magnetization rapidly increases at low fields $(H \leq 0.5 \mathrm{~T})$ for the $\alpha$ phase (Fig. 5a), and remains nearly field-independent at higher fields, up to $7 \mathrm{~T}$. In the $\beta$ phase (Fig. 5b-c), the overall magnetization values are nearly four times larger than those in the $\alpha$ phase. Given 


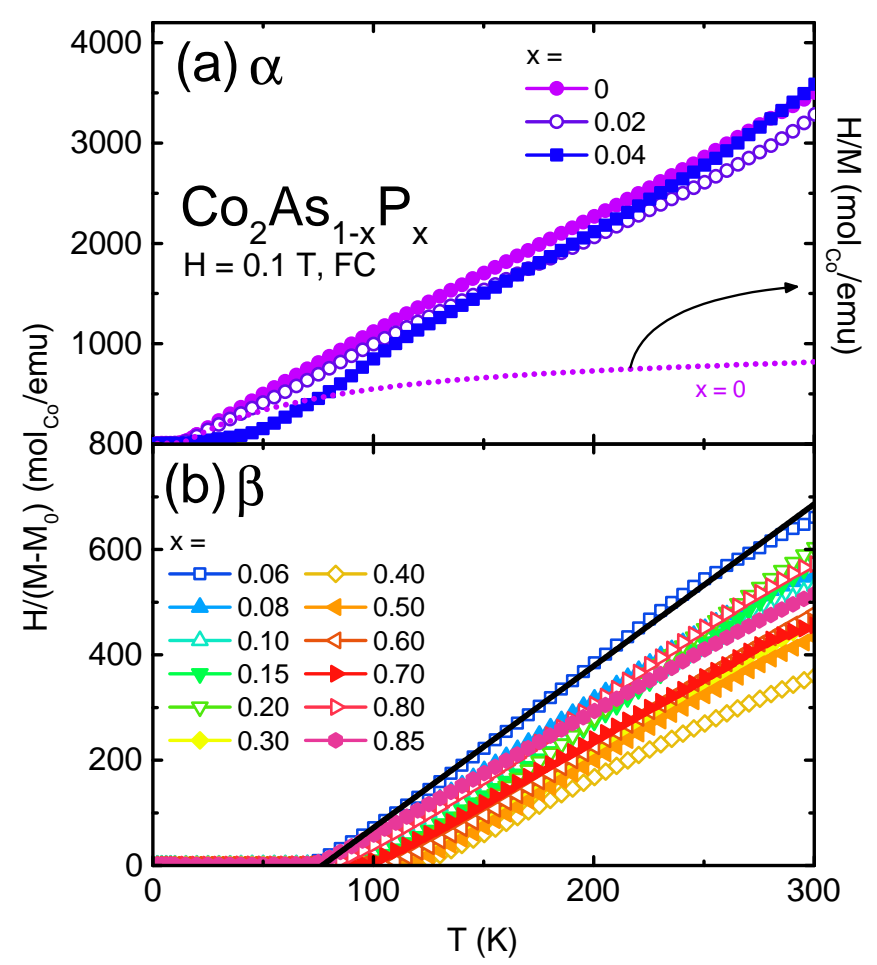

FIG. 4: Temperature-dependent inverse susceptibility for (a) $0 \leq x \leq 0.04$ ( $\alpha$ ), (b) $0.04 \leq x \leq 0.85$ ( $\beta$ ), indicating CurieWeiss-like behavior up to $300 \mathrm{~K}$. The dotted line in (a) shows the $x=0$ inverse susceptibility before subtracting a temperatureindependent constant $M_{0} / H$. The solid line in (b) illustrates a linear fit in the paramagnetic state.

the small slope in the $M(H)$ data close to the maximum applied field $H=7 \mathrm{~T}$, it is reasonable to assume that, for $x \leq 0.85$, the saturated moment $\mu_{\text {sat }}$ is only slightly higher than $M(7 \mathrm{~T})$. One can therefore approximate $\mu_{\text {sat }}$ in the hexagonal phase with the highest measured magnetization $\mu_{s a t} \approx M(7 \mathrm{~T})$. This yields $\mu_{\text {sat }}$ values between 0.06 and $0.08 \mu_{\mathrm{B}} /$ Co for the $\alpha$ phase. In the $\beta$ phase, the saturated moment first increases with $x$ from $\sim 0.33$ $\mu_{\mathrm{B}} /$ Co for $x=0.06$ to $\sim 0.43 \mu_{\mathrm{B}} /$ Co for $x=0.4$. Further $\mathrm{P}$ doping decreases the saturated moment towards $\sim$ $0.29 \mu_{\mathrm{B}} /$ Co at $x=0.85$. These saturated moment values in the $\beta$ phase $(0.06 \leq x \leq 0.85)$ are consistent with those reported previously. ${ }^{4}$ In the orthorhombic phase $(0.95 \leq x \leq 1)$, the field dependence of the magnetization reveals paramagnetic behavior (Fig. 5d). The overall magnetization values become nearly two orders of magnitude smaller than those for the hexagonal phase, and vary linearly with $H$ up to the maximum applied field $H=7 \mathrm{~T}$.

The correlation between the magnetic and electrical transport properties of $\mathrm{Co}_{2} \mathrm{As}_{1-x} \mathrm{P}_{x}$ was studied by DC resistivity measurements. The weak temperature dependence of the resistivity in the paramagnetic state and the large $\rho$ values (between 200 and $1200 \mu \Omega \mathrm{cm}$ ) indicate poor metallic behavior for all compositions (Fig. 6). For $0 \leq x \leq 0.85$, the magnetic order is signaled by a drop in resistivity consistent with the loss of spin disorder scattering. The resistivity derivatives $d \rho / d \mathrm{~T}$ are used to determine the ferromagnetic ordering temperature $\mathrm{T}_{C}$, with two examples shown in the insets in Fig. 6a-b. Large symbols in Fig. 6a-b mark the respective $T_{C}$ values for each composition in the ferromagnetic state. For the orthorhombic phase, no features were observed in the resistivity between 2 and 300 K (Fig. 6c), consistent with the suppression of the magnetic order, as suggested earlier by magnetic susceptibility 


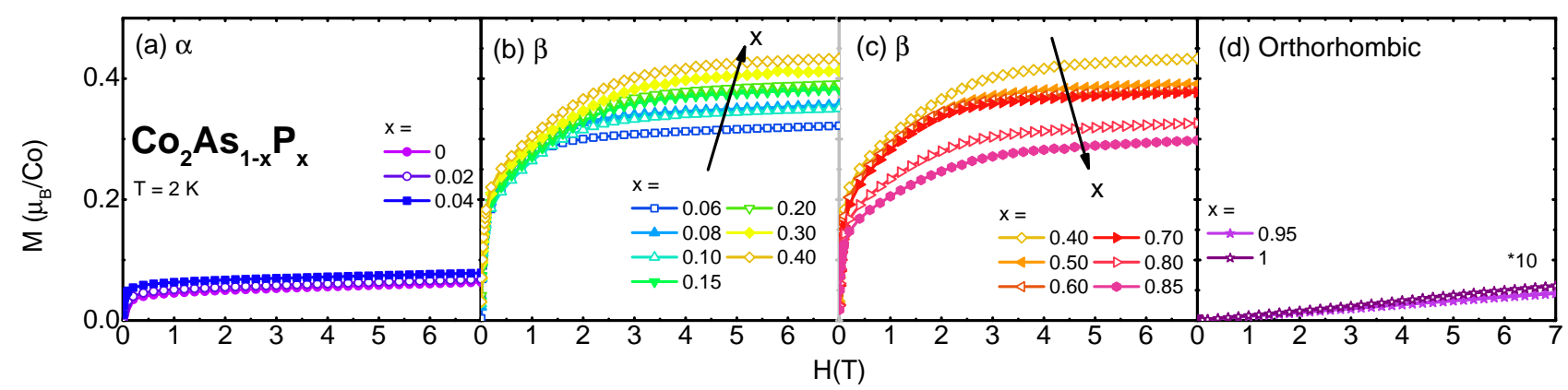

FIG. 5: $M(H)$ for (a) $0 \leq x \leq 0.04$ ( $\alpha$ phase), (b) $0.06 \leq x \leq 0.4$ ( $\beta$ phase), (c) $0.4 \leq x \leq 0.85$ ( $\beta$ phase), and (d) $0.95 \leq x \leq 1$ (orthorhombic structure). $M(H)$ in the ferromagnetic region $(0 \leq x \leq 0.85)$ increases more rapidly than in the paramagnetic region $(0.95 \leq x \leq 1)$ and saturates at $H=7 \mathrm{~T}$.

measurements (Fig. 3d). In Fig. 6a, the $x=0.04$ curve is shown as measured, while the other curves are shifted vertically by 0.05 for clarity. In (b) and (c), the $x=0.85$ and $x=1$ curves are shown as measured, while the other curves are shifted vertically from each other by 0.05 for clarity.

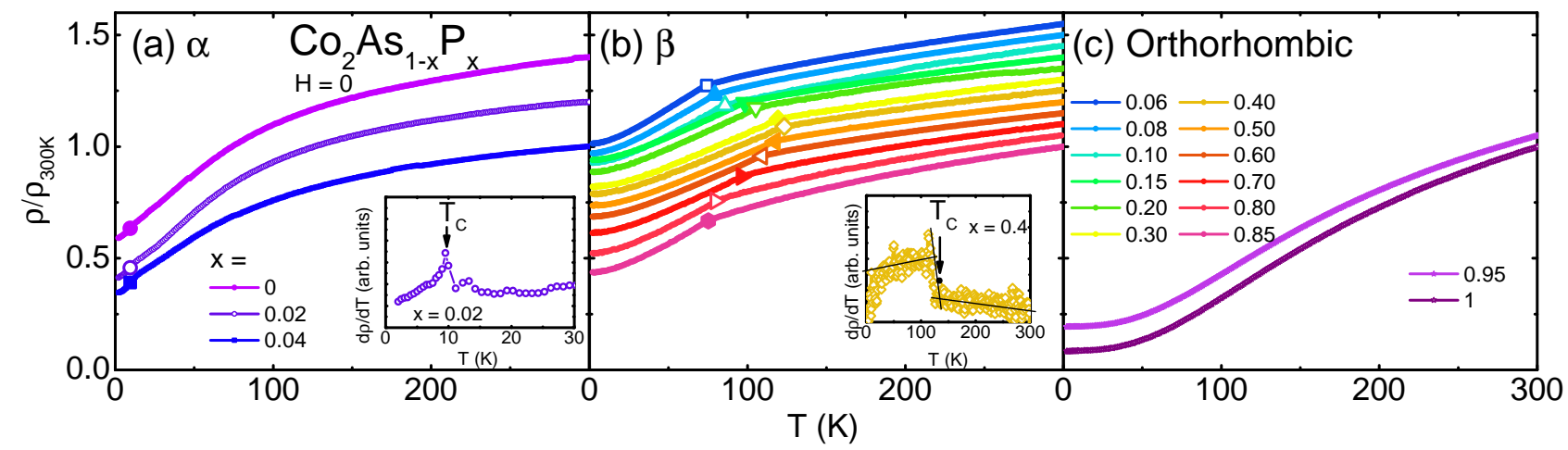

FIG. 6: $\mathrm{Co}_{2} \mathrm{As}_{1-x} \mathrm{P}_{x} H=0$ DC resistivity scaled by the $T=300 \mathrm{~K}$ values, for (a) $0 \leq x \leq 0.04$ ( $\alpha$ phase), (b) $0.06 \leq x \leq 0.85$ ( $\beta$ phase), and (c) $0.95 \leq x \leq 1$ (orthorhombic structure). The (a) $x=0.04$, (b) $x=0.85$ and (c) $x=1$ curves are shown as measured, while the other curves are shifted vertically by 0.05 for clarity. The insets in (a) and (c) indicate how $T_{C}$ (large symbols) was determined in the $\alpha$ and $\beta$ phase, respectively. The data are scaled by the resistivity at $T=300 \mathrm{~K}$.

Additional evidence for the magnetic ordering is observed in the specific heat data shown in Fig. 7. In the $\alpha$ phase $(0 \leq x \leq 0.04)$, although the magnetic transition was observed in magnetization and resistivity, only a weak feature around $T_{C}$ was evident from the specific heat measurements (Fig. 7a). However, in the $\beta$ phase $(0.06 \leq x \leq 0.85)$, the transition becomes more pronounced in specific heat, as indicated by a peak around $T_{C}$. An example is shown in the inset of Fig. 7b. Consistent with the magnetization and resistivity data, no transition was observed in the specific heat for the orthorhombic phase (Fig. 7c).

Fig. 8 illustrates how the electronic and phonon specific heat coefficients $\gamma$ and $\beta$ were determined from the linear fits of $\mathrm{C}_{p} / T\left(T^{2}\right)$ at low temperatures. The $\gamma$ values change discontinuously at both structural phase transitions, with the highest values corresponding to the $\beta$ phase (squares, left axis, Fig. 11a). Within the $\beta$ phase, a minimum in $\gamma$ occurs around $x=0.4$, close to the composition with the highest ordering temperature $T_{C}$ and Weiss temperatures 


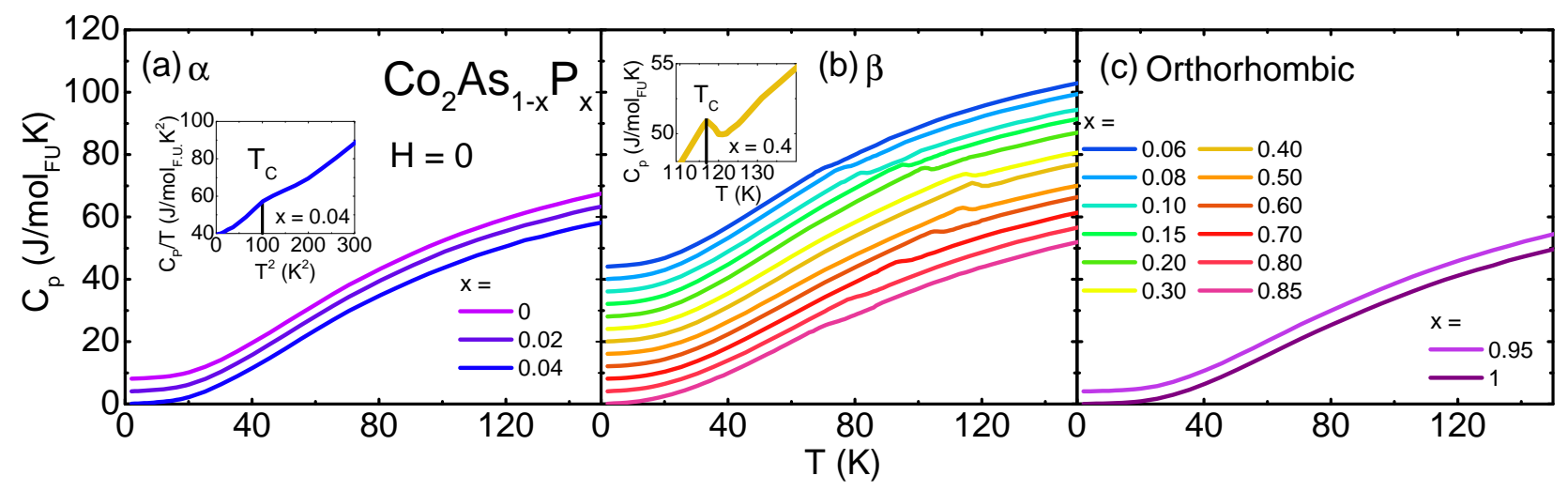

FIG. 7: The zero-field specific heat data for $\mathrm{Co}_{2} \mathrm{As}_{1-x} \mathrm{P}_{x}$ : (a) $0 \leq x \leq 0.04$ ( $\alpha$ phase), (b) $0.06 \leq x \leq 0.85$ ( $\beta$ phase), and (c) $0.95 \leq x \leq 1$ (orthorhombic structure). The $x=0.04,0.85$, and 1 curves are shown as measured, while the other curves are shifted vertically from each other by $4 \mathrm{~J} / \mathrm{mol}_{F U} \mathrm{~K}$ to avoid overlap. The inset in (a) indicates how to determine $T_{C}$ and the weak feature around $T_{C}$. The feature is so weak that can only be observed in Cp vs. $T^{2}$ plot. The inset in (b) indicates how to determine $T_{C}$ and cusps were observed in the $\beta$ phase ferromagnetic region $(0.06 \leq x \leq 0.85)$.

$\theta_{W}$ (open and full symbols, Fig. 11b).

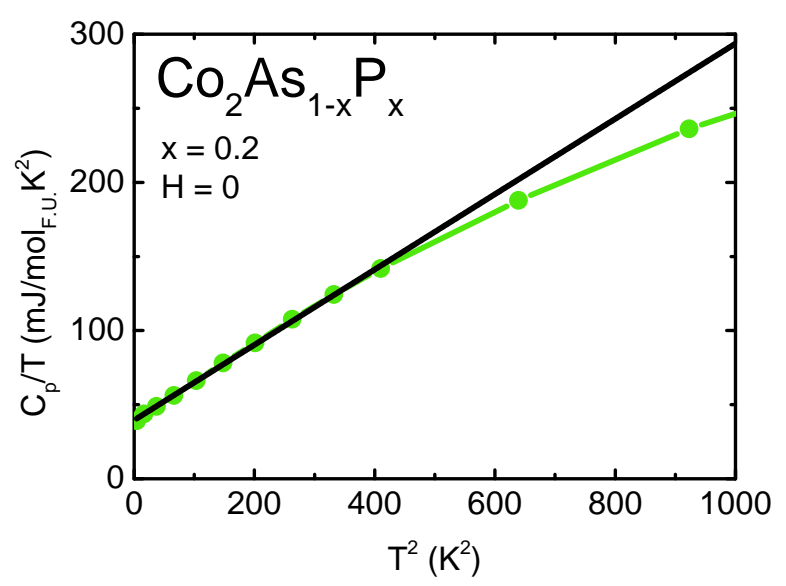

FIG. 8: A fitting was applied to $\mathrm{Co}_{2} \mathrm{As}_{0.8} \mathrm{P}_{0.2}$ to derive its $\gamma$ value.

\section{BAND STRUCTURE CALCULATIONS}

To reveal the connection between the magnetic and the structural phase transitions, band structure calculations were performed for all three phases for both PM and FM states. The PM density of states (DOS) plots for $\alpha-\mathrm{Co}_{2} \mathrm{As}$, $\beta-\mathrm{Co}_{2} \mathrm{As}$ and $\mathrm{Co}_{2} \mathrm{P}$ are shown in Fig. 9a,b and c, respectively. There are several notable features in the DOS plot. Firstly, for all three phases, the main contributions to the total DOS (black lines) close to the Fermi level are from Co ions (red lines). A similar behavior was also observed in the parent compounds for the Fe-pnictide superconductors, e.g. $\mathrm{LaFeAsO}^{22}$, for which the electronic state close to the Fermi level can be approximately described as 2D metallic sheets of $\mathrm{Fe}^{2+}$ ions embedded in a background of anions. The band structure calculations for our compounds support 
the itinerant magnetism scenario, as was the case for Fe pnictides. The FM state calculations (Fig. 10) show that the Co atoms in all three phases have the tendency towards fully spin-polarized states, since almost all DOS contributions come from states below the Fermi level. Secondly, the PM d bands for $\alpha-\mathrm{Co}_{2} \mathrm{As}, \beta-\mathrm{Co}_{2} \mathrm{As}$ and $\mathrm{Co}_{2} \mathrm{P}$ are very narrow, as shown by the blue lines in Fig. 9a,b and c. For the $\alpha, \beta$ and orthorhombic phases, the d bandwidths are $\sim 3.39 \mathrm{eV}$, $3.25 \mathrm{eV}$ and $3.89 \mathrm{eV}$, respectively, narrower than other transition metal pnictide compounds, e.g. LaFeAsO. ${ }^{22}$ (with $\mathrm{d}$ bandwidth $\sim 4.2 \mathrm{eV}$ ). Considering that narrower bands usually imply stronger tendency towards magnetism, ${ }^{24,25}$ this trait is consistent with the experimental observation of enhanced ferromagnetism in the $\beta$ phase, for which the $\mathrm{d}$ band is the narrowest of the three phases. Thirdly, the DOS at the Fermi level $\mathrm{N}\left(\mathrm{E}_{\mathrm{F}}\right)$ for the $\alpha, \beta$ and orthorhombic phases is 3.2, 5.6 and 4.7 states $\mathrm{eV}^{-1}$ F.U. ${ }^{-1}$, respectively. According to Stoner theory, ${ }^{18,19}$ higher $\mathrm{N}\left(\mathrm{E}_{\mathrm{F}}\right)$ is associated with stronger tendency towards magnetism, which also makes $\beta-\mathrm{Co}_{2} \mathrm{As}$ most favorable towards spin polarization.

\begin{tabular}{c||c|c|c|c}
\hline Atom & $\mathrm{x}$ & $\mathrm{y}$ & $\mathrm{z}$ & Moment $\left(\mu_{B}\right)$ \\
\hline Co1 & 0.4621 & 0.6308 & 0 & 0.79 \\
Co2 & 0.8725 & 0.8725 & 0 & 0.30 \\
Co3 & 0.3749 & 0.3749 & 0 & 0.60 \\
Co4 & 0.5406 & 0.8239 & $1 / 2$ & 0.23 \\
Co5 & 0.6939 & 0.6939 & $1 / 2$ & -0.48 \\
Co6 & 0.203 & 0.203 & $1 / 2$ & 1.21 \\
\hline Co1 & 0.747 & 0.747 & 0 & 0.22 \\
Co2 & 0.408 & 0.408 & $1 / 2$ & 0.96 \\
\hline
\end{tabular}

TABLE I: Magnetic moments for different crystallographic sites for $\alpha-\mathrm{Co}_{2} \mathrm{As}$ (upper) and $\beta$-Co $\mathrm{Co}_{2}$ (lower). Negative sign indicates spin direction opposite to the direction of overall magnetization in the unit cell.

A further verification of the tendency towards magnetic order is provided by the calculations of the energies for the $\mathrm{FM}$ state. For $\alpha-\mathrm{Co}_{2} \mathrm{As}, \beta-\mathrm{Co}_{2} \mathrm{As}$ and $\mathrm{Co}_{2} \mathrm{P}$, the $\mathrm{FM}$ energies (relative to the PM state) $\Delta E(F M)=E(F M)-E(P M)$ are about $-41 \mathrm{meV} / \mathrm{F} . \mathrm{U} .,-78 \mathrm{meV} / \mathrm{F} . \mathrm{U}$. and $-4.4 \mathrm{meV} / \mathrm{F} . \mathrm{U}$. , respectively. The energy comparison clearly shows that the magnetic polarization is strongest for $\beta-\mathrm{Co}_{2} \mathrm{As}$, consistent with the experimental observation. It should be pointed out that, although in $\mathrm{Co}_{2} \mathrm{P}$ the energy for the $\mathrm{FM}$ state is lower than $\mathrm{PM}$ state, the $-4.4 \mathrm{meV} / \mathrm{F}$.U. energy difference is within the $10 \mathrm{meV}$ error bar for the FP-LAPW method. Therefore, the band structure calculations indicate that the magnetism in $\mathrm{Co}_{2} \mathrm{P}$, if present, would be very weak. Although the magnetic energy $\Delta E(F M)$ for $\alpha-\mathrm{Co}_{2} \mathrm{As}$ is roughly half of that for $\beta-\mathrm{Co}_{2} \mathrm{As}$, the Curie temperature for the former is much lower than that of the latter phase, possibly due to the presence of strong spin fluctuations in $\alpha-\mathrm{Co}_{2} \mathrm{As}$, which is supported by the deviation from Curie-Weiss law of the magnetic susceptibility (Fig. 4). A remarkable observation is that different Co sites tend to accommodate magnetic moments with different magnitudes, as summarized in Table I. For example in $\beta-\mathrm{Co}_{2} \mathrm{As}$, the magnetic moments for the $\mathrm{Co} 1$ and $\mathrm{Co} 2$ sites are $0.22 \mu_{B}$ and $0.96 \mu_{B}$.

The FM spin-polarized DOS plots for $\alpha-\mathrm{Co}_{2} \mathrm{As}, \beta-\mathrm{Co}_{2} \mathrm{As}$ and $\mathrm{Co}_{2} \mathrm{P}$ are shown in Fig. 10. For $\alpha-\mathrm{Co}_{2} \mathrm{As}$, the $\mathrm{d}$ bands for spin up and spin down channels are slightly shifted in energy, with an almost fully-filled spin-up component of the d band (with almost no spin-up d-state above Fermi energy). The small exchange splitting is consistent with the weak FM (WFM) observed experimentally. For $\beta-\mathrm{Co}_{2} \mathrm{As}$, the splitting between the spin-up and spin-down components of the $\mathrm{d}$ band is more pronounced, with the splitting energy close to $0.3 \mathrm{eV}$. Remarkably, the spin-up component of 
the $\mathrm{d}$ band is almost fully filled, with $\mathrm{E}_{F}$ at an almost zero local minimum. The asymmetry between the spin-up and spin-down $\operatorname{DOS}\left(\mathrm{E}_{\mathrm{F}}\right)$ implies that the charge carriers are strongly polarized, i.e. that most of the charge carriers ( 94\%) would be spin-down. In the case of $\mathrm{Co}_{2} \mathrm{P}$, although there is also a slight splitting between the spin-up and spin-down components of the d band, the FM state is only slightly lower in energy compared to the PM state discussed above. Summing up the spin-up and spin-down components of the $\operatorname{DOS}\left(\mathrm{E}_{\mathrm{F}}\right)$ leads to total spin-polarized DOS(EF of 3.5, 4.6 and 3.54 states $\mathrm{eV}^{-1}$ F.U. ${ }^{-1}$ for $\alpha-\mathrm{Co}_{2} \mathrm{As}, \beta-\mathrm{Co}_{2} \mathrm{As}$ and $\mathrm{Co}_{2} \mathrm{P}$, respectively. Such trend is consistent with the experimental observations that the electronic specific heat coefficient is biggest for $\beta-\mathrm{Co}_{2} \mathrm{As}$, as seen in Fig. $11 \mathrm{a}$ (full symbols, left axis).

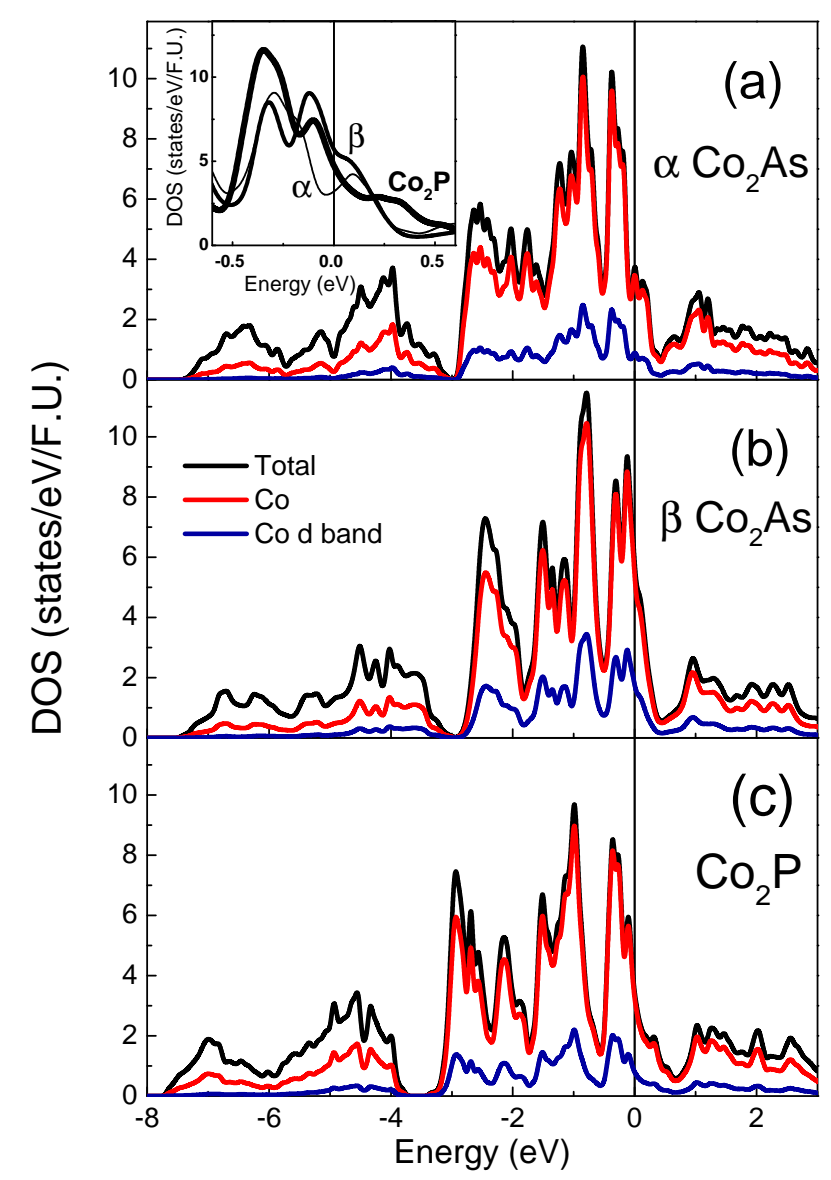

FIG. 9: The non-magnetic (PM) DOS for (a) $\alpha-\mathrm{Co}_{2} \mathrm{As}$, (b) $\beta-\mathrm{Co}_{2}$ As and (c) $\mathrm{Co}_{2} \mathrm{P}$, normalized to one formula unit. Black, red and blue lines are for total, Co, and d-band DOS, respectively. Inset in (a) shows the zoom in plot for total DOS close to the Fermi level.

\section{DISCUSSION}

The magnetic properties of $\mathrm{Co}_{2} \mathrm{As}_{1-x} \mathrm{P}_{x}$ compounds are significantly affected by the crystal structure. The $T_{C}$ and saturated moment values at $2 \mathrm{~K}$ are enhanced by the occurrence of a structural phase transition between $x$ $=0.04$ and 0.06 (Fig. 3 and Fig. 5). With Curie-Weiss-like behavior of the magnetic susceptibility in the hexagonal 


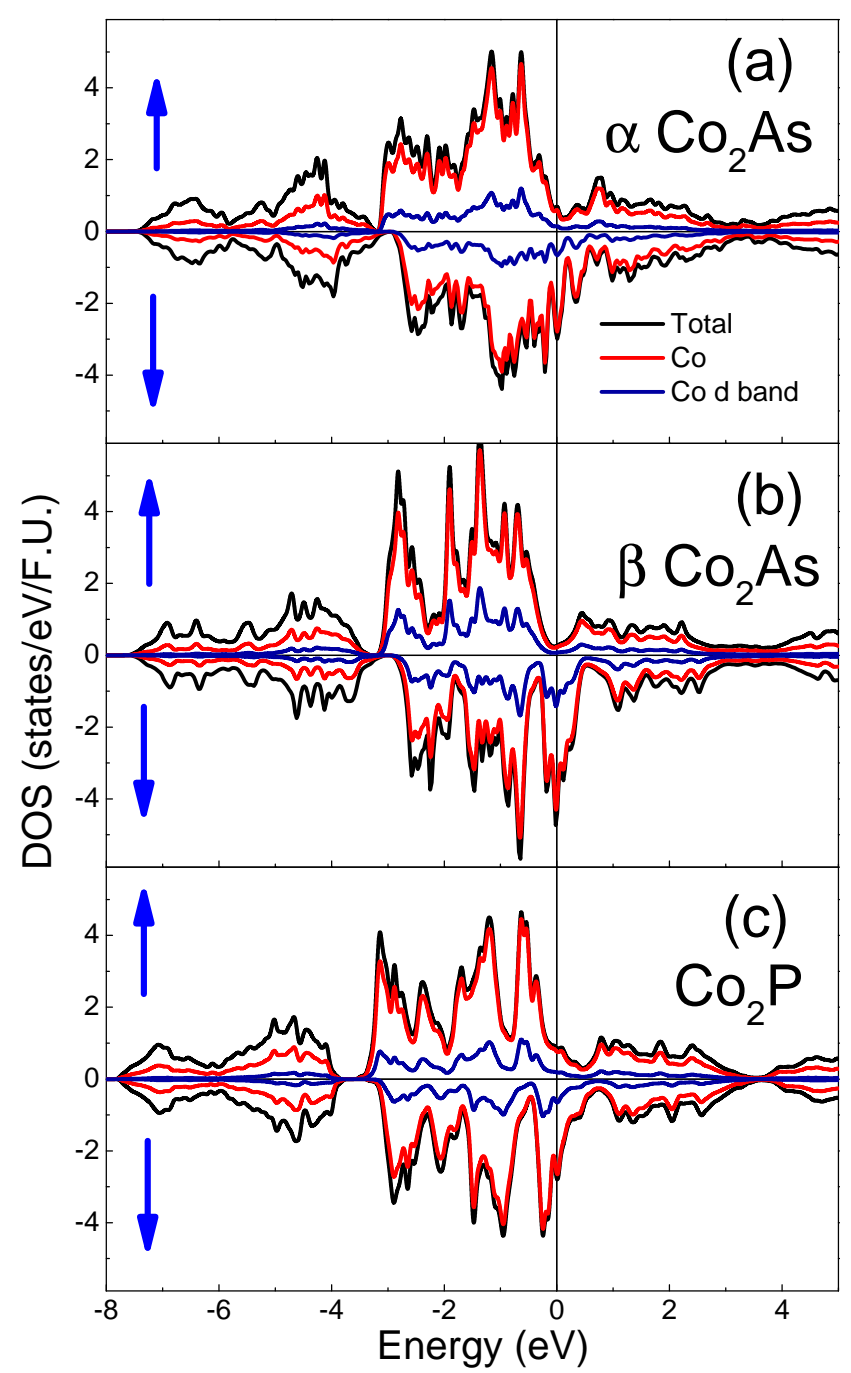

FIG. 10: The spin-polarized (FM) DOS for (a) $\alpha-\mathrm{Co}_{2} \mathrm{As}$, (b) $\beta-\mathrm{Co}_{2} \mathrm{As}$ and (c) $\mathrm{Co}_{2} \mathrm{P}$, normalized to one formula unit. Black, red and blue lines are for total, Co, and d-band DOS, respectively. The spin-down components of the DOS are multiplied by -1 for better visualization.

phase, the corresponding Weiss temperatures $\theta_{W}$ are similarly enhanced in the $\beta$ phase, with a maximum around $x$ $=0.4$. Another structural phase transition that occurs between $x=0.85$ and $x=0.95$ results in the suppression of the ferromagnetic order, to a Pauli paramagnetic state (Fig. 3 and Fig. 5). The saturated moments $\mu_{\text {sat }}=q_{s}$ in the $\alpha$ phase are between 0.06 and $0.08 \mu_{B} /$ Co (Fig. 5), about an order of the magnitude smaller than those of Co ${ }^{2+}$ and $\mathrm{Co}^{3+}$ low spin states. Because these values are much smaller than the theoretical values, it suggests that the magnetic moments are likely itinerant instead of local in the $\alpha$ phase. However, in the $\beta$ phase, the saturated magnetic moments are considerably higher, between 0.29 and $0.43 \mu_{B} /$ Co (Fig. 5). These values lie between the low spin moments for $\mathrm{Co}^{2+}$ and $\mathrm{Co}^{3+}$, suggesting two plausible scenarios for the origin of magnetism. One scenario consists of a mixed valence system with $\mathrm{Co}^{2+}$ and $\mathrm{Co}^{3+}$ local moments while the other draws on the itinerant moment picture. To distinguish between the local moment and itinerant moment scenarios in $\mathrm{Co}_{2} \mathrm{As}_{1-x} \mathrm{P}_{x}$, the Rhodes-Wohlfarth ratio was calculated. ${ }^{20}$

For estimating the Rhodes-Wohlfarth ratio $\mathrm{q}_{c} / \mathrm{q}_{s}, q_{c}$ is estimated from the paramagnetic susceptibility as $p_{e f f}$ 
$=\sqrt{q_{c}\left(q_{c}+2\right)}$, and $q_{s}$ is the saturated moment $q_{s}=\mu_{s a t}$, where $p_{\text {eff }}$ is the effective moment and $\mu_{\text {sat }}$ is the saturated moment. ${ }^{17,20}$ For local moment magnets, the Rhodes-Wohlfarth ratio is close to 1 , but it diverges in the itinerant moment limit. The Rhodes-Wohlfarth ratio for $\mathrm{Co}_{2} \mathrm{As}_{1-x} \mathrm{P}_{x}$ with a FM ground state $(0.06 \leq \mathrm{x} \leq 0.85)($ open symbols, right axis, Fig. 11a) is $\gtrsim 3$. These values suggest an itinerant moment scenario for the hexagonal states ( $\alpha$ and $\beta$ ). It should be noted that the slightly enhanced $\mathrm{q}_{c} / \mathrm{q}_{s}$ ratio in the $\alpha$ phase is likely an artifact of taking the ratio of two small numbers rather than an intrinsic increase for $\mathrm{x}<0.04$.

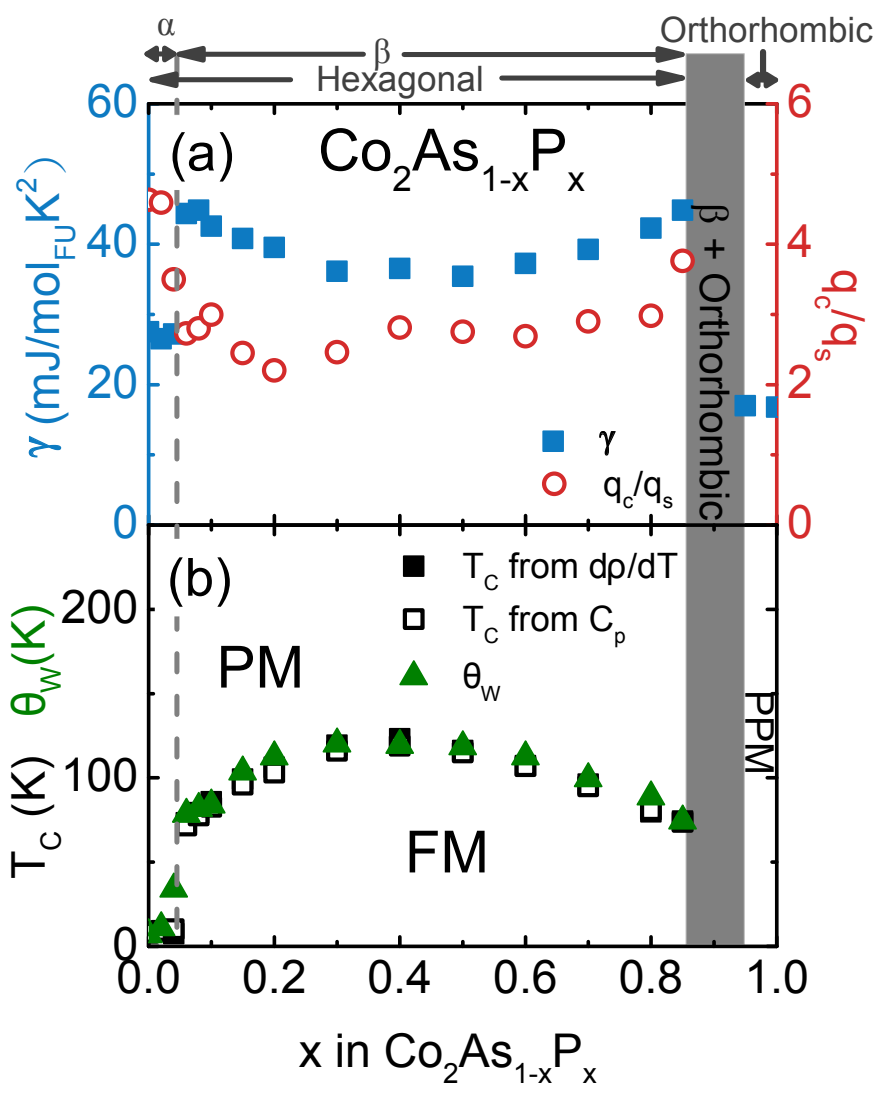

FIG. 11: a $\gamma$ values derived from specific heat data and the Rhodes-Wohlfarth ratio calculated from effective and saturated moments. Error bars are smaller than symbols. (b) Phase diagram of $\mathrm{Co}_{2} \mathrm{As}_{1-x} \mathrm{P}_{x}: T_{C}$ derived from resistivity and specific heat, and Weiss-temperature derived from the Curie-Weiss fit as a function of composition $x$. For $0 \leq x \leq 0.85$, the compounds are in the hexagonal phase and exhibit ferromagnetic behavior (FM). Above $T_{C}$, compounds show Curie-Weiss-like paramagnetism $(\mathrm{PM})$. For $0.95 \leq x \leq 1$, the compounds show Pauli paramagnetism (PPM). The gray dashed line indicates the structural phase transition between the $\alpha$ phase and the $\beta$ phase. The gray region indicates the mixed phases region.

The structural phase transitions also cause the reconstruction of the Fermi surface, suggested by the composition dependence of the electronic specific heat coefficient $\gamma$ (full symbols, left axis, Fig. 11a). In $\mathrm{Co}_{2} \mathrm{As}_{1-x} \mathrm{P}_{x}$, the hexagonal-to-orthorhombic structural transition that happens between $x=0.85$ and 0.95 causes a larger change of the $\gamma$ value than the structure transition between $x=0.04$ and 0.06 . This is consistent with the larger change of the crystal structure and the Fermi surface reconstruction between $x=0.85$ and $0.95 . \mathrm{Co}_{2} \mathrm{As}_{1-x} \mathrm{P}_{x}$ in the ferromagnetic region $(0 \leq x \leq 0.85)$ has a drop in the $\gamma$ values, such that $\gamma$ in the ferromagnetic (hexagonal $\beta)$ state $(0.06 \leq x \leq 0.85)$ is nearly three times larger than in the non-magnetic (orthorhombic) state $(0.95 \leq x \leq 1)$ or in the $\alpha$ phase $(x \leq 0.04)$. 
In the $\alpha$ and orthorhombic phases, the $\gamma$ values were nearly composition independent. By contrast, in the $\beta$ phase, $\gamma$ varies inversely with $\mu_{\text {sat }}, T_{C}$ and $\theta_{W}$ (Fig. 5 and Fig. 11b) and has a minimum at $x=0.4$. The composition dependence of $\gamma$ in this region shows that the largest $\mu_{\text {sat }}$ (for $x=0.4$ ) is associated with the smallest density of states at the Fermi surface. This is exactly opposite to the itinerant moment scenario, for which $\mu_{\text {sat }}$ should be maximized by the maximum density of state at Fermi surface. However, $\mu_{\text {sat }}$ also depends on the splitting energy of the spin-up and spin-down bands. ${ }^{24}$ Therefore, a large splitting energy in $\mathrm{Co}_{2} \mathrm{As}_{1-x} \mathrm{P}_{x}$ for $x=0.4$ could explain the maximum $\mu_{\text {sat }}$ for this composition.

Band structure calculations agree with the experimental observation: the magnetic energy is largest for $\beta$ $\mathrm{Co}_{2} \mathrm{As}$, with strong spin fluctuations in this phase suggested by the fact that, from experiment, it is found that its $\mathrm{T}_{C}$ is nearly one order of magnitude higher then that of the $\alpha$ phase (Fig. 11b, open symbols), while the calculations indicate that the magnetic energy is only twice as large as that of the $\alpha$ phase. Moreover, $\mathrm{N}\left(\mathrm{E}_{\mathrm{F}}\right)$ is highest for $\beta-\mathrm{Co}_{2} \mathrm{As}$, which is also consistent with the enhanced electronic specific heat coefficient value for the $\beta$ phase, as shown in Fig. 11a. The dominance of Co DOS in the three compounds close to the Fermi level is reminiscent of the parent compounds of Fe pnictide superconductors, which favors an itinerant nature of the magnetism. Further chemical or pressure tuning might be able to shift the balance between the itinerant magnetic ground state and competing states, possibly favoring superconductivity for some appropriate tuning parameters.

In summary, we report that the magnetic properties of $\mathrm{Co}_{2} \mathrm{As}_{1-x} \mathrm{P}_{x}$ are strongly correlated with the crystal structure. For $0 \leq x \leq 0.04$, the compounds form in the $\alpha$ phase and show a ferromagnetic ground state. The $\alpha$-to- $\beta$ structural phase transition that occurs between $\mathrm{x}=0.04$ and 0.06 results in the enhancement of ferromagnetism. The $T_{C}$ in the $\beta$ phase $(0.06 \leq x \leq 0.85)$ is an order of magnitude higher than in the $\alpha$ phase. Another structural phase transition takes place between $x=0.85$ and 0.95 , resulting in the quenching of the magnetic order. After this

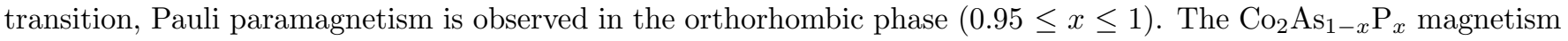
is itinerant, as suggested by the large Rhodes-Wohlfarth ratios (Fig. 11a, open symbols, right axis), observed metallic behavior (Fig. 6), and band structure calculations (Figs. 9 and 10). The enhancement of the itinerant ferromagnetic order driven solely by structural phase transitions provides a potential route for tuning the magnetism in other binary or more complex compounds.

\section{ACKNOWLEDGEMENTS}

This work was support by DOD PECASE and AFOSR MURI. We are thankful to A.H. Nevidomskyy for useful discussions.

${ }^{1}$ R. Fruchart, A. Roger, and J. P. Senateur, J. Appl. Phys. 40, 1250 (1969).

2 A. Kjekshus and K. E. Skaug, Acta Chem. Scand. 26, 2554 (1972).

3 J. Goodenough, J. Solid State Chem. 7, 428 (1973).

4 A. Krumbügel-Nylund, D. Boursier, A. Rouault, J. P. Senateur, and R. Fruchart, Mat. Res. Bull. 9, 21 (1974).

${ }^{5}$ N. Iwata, T. Matsushima, H. Fujii, and T. Okamoto, J. Phys. Soc. Jpn. 50, 729 (1981).

${ }^{6}$ G. Meisner and H. Ku, Appl. Phys. A 31, 201 (1983). 
7 S. Fujii, S. Ishidai, and S. Asanot, J. Phys. F: Met. Phys. 18, 971 (1988).

8 S. Ohta, J. Phys. Soc. Jpn. 65, 4090 (1996).

${ }^{9}$ P. Önnerud, Y. Andersson, R. Tellgren, P. Nordbladb Solid State Commun. 101, 271 (1997).

10 S. Ohta and H. Onmayashiki, Physica B 253, 193 (1998).

11 M. Artigas, M. Bacmann, D. Fruchart, R. Fruchart, J.L. Soubeyroux, and P. Wolfers, C. R. Acad. Sci., Ser. II 313, 635 (1991).

12 A. Larson and R. V. Dreele, Los Alamos National Laboratory Report LAUR 86, 748 (2004).

13 P. Blaha, K. Schwarz, G. Madsen, D. Kvasnicka, and J. Luitz, An Augmented Plane Wave + Local Orbitals Program for Calculating Crystal Properties, Vienna University of Technology, Vienna, Austria, (2001).

14 J. P. Perdew, K. Burke, and M. Ernzerhof, Phys. Rev. Lett. 77, 3865 (1996).

15 V. L. Vegard, Z. Phys. 5, 17 (1921).

16 N. W. Ashcroft and N. D. Mermin, Solid State Physics, Thomson Learning, Inc., (1976).

17 P. Rhodes and E. P. Wohlfarth, Proc. R. Soc. London, Ser. A 273, 247 (1963).

18 E. C. Stoner, Proc. R. Soc. London, Ser. A 165, 372 (1938).

19 E. C. Stoner, Proc. R. Soc. London, Ser. A 169, 339 (1939).

20 E. Wohlfarth, J. Magn. Magn. Mater. 7, 113 (1978).

21 T. Moriya, Spin Fluctuations in Itinerant Electron Magnetism, Springer-Verlag, (1985).

22 D. J. Singh and M.-H. Du, Phys. Rev. Lett., 100, 237003 (2008).

${ }^{23}$ R. A. de Groot, F. M. Mueller, P. G. van Engen, and K. H. J. Buschow, Phys. Rev. Lett., 50, 2024 (1983).

24 T. Moriya and H. Hasegawa J. Phys. Soc. Jpn. 48, 1490 (1980).

25 M.I. Auslender, V.Y. Irkhin and M.I. Katsnelson J. Phys. C: Solid State Phys. 21, 5521 (1988). 